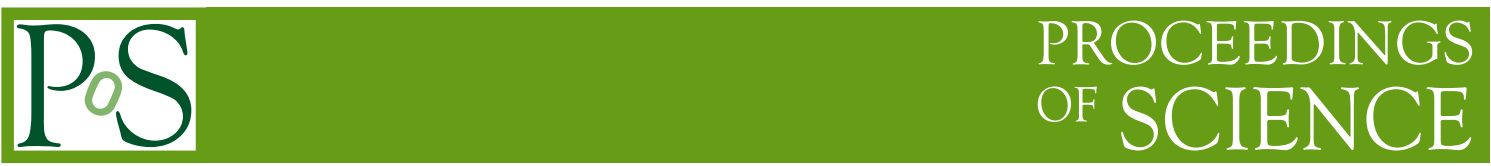

\title{
Gamma-rays from Cataclismic Variables
}

\author{
Wlodek Bednarek \\ Department of Astrophysics, University of Lodz, Lodz, Poland \\ E-mail: bednareastro.phys.uni.lodz.pl \\ Jerzy Pabich \\ Department of Astrophysics, University of Lodz, Lodz, Poland \\ E-mail: jpabic@uni.lodz.pl
}

\begin{abstract}
The accretion of matter onto intermediate polar White Dwarfs (IPWDs) seems to provide attractive conditions for acceleration of particles to high energies in a strongly magnetized turbulent region at the accretion disk inner radius. We consider possible acceleration of electrons and hadrons in such region and investigate their high energy radiation processes. It is concluded that accelerated electrons loose energy mainly on synchrotron process producing non-thermal X-ray emission. On the other hand, accelerated hadrons are convected onto the WD surface and interact with dense matter. As a result, high energy $\gamma$-rays from decay of neutral pions and secondary leptons from decay of charged pions appear. We show that GeV-TeV $\gamma$-rays can escape from the vicinity of the WD. Secondary leptons produce synchrotron radiation in the hard X-rays and soft $\gamma$-rays. As an example, we predict the X-ray and $\gamma$-ray emission from IPWD V1223 Sgr. Depending on the spectral index of injected particles, this high energy emission may be detected by the Fermi-LAT telescope and/or the future Cherenkov Telescope Array (CTA) observatory.
\end{abstract}

25th Texas Symposium on Relativistic Astrophysics - TEXAS 2010

December 06-10, 2010

Heidelberg, Germany 


\section{Introduction}

Intermediate polar Cataclismic Variables (CVs) have been recently established by the INTEGRAL as a class of the hard X-ray sources with evidences of non-thermal components (e.g. Barlow et al. 2008; Revnivtsev et al. 2008). The physical processes in Cataclysmic Variables are expected to be similar to those observed in the $\mathrm{X}$-ray binaries containing accreting neutron stars. Therefore, $\mathrm{CVs}$ were also suspected to be sites of high energy processes. Unfortunately, none of such objects has been detected in the $\gamma$-rays by the EGRET telescope (Schlegel et al. 1995). However, a sporadic $\mathrm{TeV} \gamma$-ray emission has been reported by two independent collaborations from the AE Aqr which accretes matter in the propeller phase (Meintjes et al. 1992; 1994; Bowden et al. 1992; Chadwick et al. 1995). More recent observations of this source by the Whipple and the MAGIC telescopes do not report any evidence for steady or pulsed emission (Lang et al. 1998; Sidro et al. 2008). Also a polar type CV, AM Her, has been reported as a TeV $\gamma$-ray source by Bhat et al. (1991). TeV $\gamma$-ray observations of AE Aqr and AM Her have stimulated investigation of different scenarios for the $\gamma$-ray production in CVs. They are mainly modifications of the models proposed for the high energy processes in accreting neutron stars. Recently, we have discussed a model in which particles (electrons, hadrons) are accelerated in a turbulent, strongly magnetized transition region between rotating magnetosphere of the neutron star and the accretion disk (Bednarek 2009, for the earlier version see Tavani \& Liang 1993). We show that for the conditions expected in the IPWDs, particles can also reach energies suitable for the hard $\mathrm{X}$-ray and $\mathrm{GeV}-\mathrm{TeV} \gamma$-ray production.

\section{Accretion onto White Dwarf}

White Dwarfs (WD), accreting in the accretor phase, have typical magnetic momenta twothree orders of magnitude larger then the neutron stars due to significantly larger radii. Therefore, the accretion process is influenced by the magnetic field typically at larger distances from the WD than in the case of neutron stars. The accretion rate of matter onto the WD surface, $\left(\dot{M}_{\text {acc }}=10^{17} M_{17} \mathrm{~g} \mathrm{~s}^{-1}\right)$, can be estimated from the observed thermal X-ray emission, $L_{\mathrm{X}} \cdot \dot{M}_{\text {acc }}$ and $L_{\mathrm{X}}$ can be related to the known radius and mass of the WD (we assume $R_{\mathrm{WD}} \approx 5 \times 10^{8} \mathrm{~cm}$ and $\left.M_{\mathrm{WD}}=0.9 M_{\odot}\right), L_{\mathrm{x}}=G M_{\mathrm{WD}} \dot{M} / R_{\mathrm{WD}} \approx 2.4 \times 10^{34} M_{17} \quad \mathrm{erg} \mathrm{s}^{-1}$. Due to the strong magnetic field of rotating WD, the pressure of the accreting matter is balanced by the pressure of the rotating magnetosphere. The distance, at which the magnetic field begins to dominate the dynamics of matter (so called the Alfven radius), can be estimated by comparing the magnetic-field energy density with the kinetic-energy density of matter, $B_{\mathrm{A}, \mathrm{o}}^{2} / 8 \pi=\rho v_{\mathrm{f}}^{2} / 2$, where $B_{\mathrm{A}, \mathrm{o}}$ is the magnetic field strength in the inner magnetosphere of the $\mathrm{WD}, \rho=\dot{M}_{\mathrm{acc}} /\left(\pi R_{\mathrm{A}, \mathrm{O}}^{2} v_{\mathrm{f}}\right)$ is the density of accreting matter, $v_{\mathrm{f}}=\left(2 G M_{\mathrm{WD}} / R_{\mathrm{A}, \mathrm{O}}\right)^{1 / 2}$ is the free fall velocity of accreting matter, $R_{\mathrm{A}, \mathrm{o}}$ is the Alfven radius in the case of the spherically-symmetric accretion, and $G$ is the gravitational constant. The medium in the transition region is very turbulent and strongly magnetized, providing good conditions for the acceleration of particles to high energies. We estimate the location of this region from the surface of the WD assuming that the magnetic field in the inner WD magnetosphere is of the dipole type, i.e. $B_{\mathrm{A}}=B_{\mathrm{WD}}\left(R_{\mathrm{WD}} / R_{\mathrm{A}}\right)^{3}$, then, $R_{\mathrm{A}, \mathrm{o}}=3.5 \times 10^{9} B_{6}^{4 / 7} M_{17}^{-2 / 7} \mathrm{~cm}$, where the magnetic field at the WD surface is $B_{\mathrm{WD}}=10^{6} B_{6}$ G. However, in the case of a disk accretion, the location of the Alfven radius is much more difficult to estimate since it depends on the details of the 
accretion process through the disk. In general, the Alfven radius, $R_{\mathrm{A}}$, can be related to $R_{\mathrm{A}, \mathrm{o}}$ by applying the scaling factor, $\chi$, which lays approximately in the range $\sim 0.1-1, R_{\mathrm{A}}=\chi R_{\mathrm{A}, \mathrm{o}}$. Based on the known value of $R_{\mathrm{A}}$, we can estimate the magnetic field strength at the transition region, $B_{\mathrm{A}}=2.9 \times 10^{3} \chi^{-3} M_{17}^{6 / 7} B_{6}^{-5 / 7} \quad$ G. The accretion of matter onto WD can occur provided that the rotational velocity of the magnetosphere at $R_{\mathrm{A}}$ is lower than the Keplerian velocity of the accreting matter. The rotational velocity is, $v_{\text {rot }}=2 \pi R / P$, and the Keplerian velocity is $v_{\mathrm{k}}=\left(G M_{\mathrm{WD}} / R\right)^{1 / 2}$, where $R$ is the distance from the center of the WD. The distance at which $v_{\mathrm{rot}}=v_{\mathrm{k}}$ is called the corotation radius, $R_{\mathrm{co}} \approx 3.1 \times 10^{9} P_{2}^{2 / 3} \mathrm{~cm}$, where the WD period $P=10^{2} P_{2} \mathrm{~s}$. When $R_{\mathrm{A}}>R_{\mathrm{co}}$ then accretion occurs in the propeller regime. In the opposite case, $R_{\mathrm{A}}<R_{\mathrm{co}}$, i.e. for the rotational periods of the WDs fulfilling the condition, $P>120 \chi^{3 / 2} B_{6}^{6 / 7} M_{17}^{-3 / 7} \mathrm{~s}$, the accretion process occurs in the accretor phase. Here we are mainly interested in the accretor phase, since in this case we can get information on the accretion rate onto the WD from the observed X-ray luminosity produced close to the WD surface.

It is expected that in the case of accretion process through the formation of an accretion disk, matter can penetrate closer to the surface of the WD. We can estimate density of matter at the disk inner radius as a function of the parameter, $\chi$, by using the balance equation, $B_{\mathrm{A}}^{2} / 8 \pi=\rho v_{\mathrm{k}}^{2} / 2$. By reversing this equation, we estimate density of matter at the disk inner radius on, $\rho \approx 1.2 \times$ $10^{13} \chi^{-5} B_{6}^{-6 / 7} M_{17}^{10 / 7} \mathrm{~cm}^{-3}$.

\section{Production of radiation}

In the conditions expected for the transition region (a strongly magnetized and turbulent medium) between the inner WD magnetosphere and the accreting flow, particles should be efficiently accelerated. The energy gain rate of particles with energy $E$ (and the Lorentz factor $\gamma$ ) is often parametrized by the Larmor radius of electrons and the so-called acceleration parameter, $\dot{P}_{\text {acc }}=\xi_{c E} / r_{\mathrm{L}} \approx 4.2 \times 10^{4} \chi^{-3} \xi_{17}^{6 / 7} B_{6}^{-5 / 7} \mathrm{erg} \mathrm{s}^{-1}$, where $c$ is the velocity of light, $r_{\mathrm{L}}=E / e B_{\mathrm{A}}$ is the Larmor radius, $B_{\mathrm{A}}$ is the magnetic field strength in the acceleration region, $e$ is the electron charge and $\xi$ is the acceleration parameter. We estimate the order of magnitude value for $\xi$ at the transition region on, $\xi \sim \beta\left(v_{\mathrm{k}} / c\right)^{2} \approx 3.8 \times 10^{-5} \beta \chi^{-1} M_{17}^{2 / 7} B_{6}^{-4 / 7}$. where $\beta \leq 1$ is the factor which describes the efficiency of acceleration. Then, the energy gains of particles can be expressed by, $\dot{P}_{\text {acc }} \approx 1.6 \beta \chi^{-4} M_{17}^{8 / 7} B_{6}^{-9 / 7} \mathrm{erg} \mathrm{s}^{-1}$. In principle, both electrons and hadrons can be accelerated in such a transition region. During the acceleration process, electrons also experience energy losses due to the synchrotron radiation and the Inverse Compton Scattering (ICS) of radiation from the companion star, the accretion disk, and the surface of the WD. These energy losses determine the maximum energies of accelerated electrons. The gravitational energy of accreting matter, generated in the accretion disk, can be estimated from $L_{\mathrm{D}}=\frac{G M_{\mathrm{wD}} \dot{M}}{R_{\mathrm{A}}} \approx 3.4 \times 10^{33} \chi^{-1} B_{6}^{-4 / 7} M_{17}^{9 / 7} \quad \frac{\mathrm{erg}}{\mathrm{s}}$. In the case of a simple model of the accretion disk (Shakura \& Sunyaev 1973), the disk luminosity can be related to its inner radius and the temperature at the inner radius by $L_{\mathrm{D}}=4 \pi R_{\mathrm{A}}^{2} \sigma_{\mathrm{SB}} T_{i n}^{4}$. Then, the temperature at the disk inner radius is, $T_{\text {in }}=\left(\frac{L_{\mathrm{D}}}{4 \pi R_{\mathrm{A}}^{2} \sigma_{\mathrm{SB}}}\right)^{1 / 4} \approx 2.5 \times 10^{4} \frac{\chi^{-3 / 4} M_{17}^{13 / 28}}{B_{6}^{12 / 28}} \mathrm{~K}$. Electrons lose energy with the ICS process in the Thomson $(\mathrm{T})$ and the Klein-Nishina $(\mathrm{KN})$ regimes. We estimate the photon energy density at the acceleration region (the transition region at the inner disk 
radius) to be, $\rho_{\mathrm{r}}=\frac{4 \sigma_{\mathrm{SB}} T_{\mathrm{in}}^{4}}{c} \approx 3 \times 10^{3} \chi^{-3} M_{17}^{13 / 7} B_{6}^{-12 / 7} \mathrm{erg} \mathrm{cm}^{-3}$, where $\sigma_{\mathrm{SB}}$ is the Stefan-Boltzmann constant.

We also estimate the energy density of the magnetic field at the transition region $\left(R_{\mathrm{A}}\right.$ to be, $\rho_{\mathrm{B}}=B_{\mathrm{A}}^{2} / 8 \pi \approx 3.3 \times 10^{5} \chi^{-6} M_{17}^{12 / 7} B_{6}^{-10 / 7} \mathrm{erg} \mathrm{cm}^{-3}$. The energy density of the magnetic field dominate over the energy density of radiation in the transition region, except some limitting cases when the parameter $\chi$ is close to 0.1 . We do not consider such extreme model parameters here concentrating on the cases when $\chi$ is clearly above 0.1 .

The energy losses of electrons due to each of the considered process (synchrotron and IC in the $\mathrm{T}$ regime) can be calculated from, $\dot{P}_{\text {loss }}=(4 / 3) c \sigma_{\mathrm{T}} \rho \gamma^{2} \approx 2.7 \times 10^{-14} \rho_{\mathrm{B}} \gamma^{2} \mathrm{erg} \mathrm{s}^{-1}$, where $\sigma_{\mathrm{T}}$ is the Thomson cross section. The maximum energies of accelerated electrons are determined by the balance between the energy gains from the acceleration process and the energy losses due to synchrotron processes, $\gamma_{\mathrm{e}}^{\max } \approx 1.35 \times 10^{4} \beta^{1 / 2} \chi B_{6}^{1 / 14} M_{17}^{-2 / 7}$. Based on the above formula, we can estimate the maximum energies of synchrotron photons produced by these electrons at the transition region on, $\varepsilon_{\max } \approx m_{\mathrm{e}}\left(B_{\mathrm{A}} / B_{\mathrm{cr}}\right)\left(\gamma_{\mathrm{e}}^{\max }\right)^{2} \approx 160 \xi \mathrm{MeV}$, It is of the order of $\varepsilon_{\max } \sim 6 \beta \chi^{-1} M_{17}^{2 / 7} B_{6}^{-4 / 7}$ $\mathrm{keV}$.

Energy loss rate of relativistic hadrons in interactions with matter on pion production can be estimated from, $\dot{P}_{\mathrm{pp}}=\sigma_{\mathrm{pp}} c \rho K m_{\mathrm{p}} \gamma_{\mathrm{p}} \approx 8 \times 10^{-6} \chi^{-5} B_{6}^{-6 / 7} M_{17}^{10 / 7} \gamma_{\mathrm{p}} \frac{\mathrm{erg}}{\mathrm{s}}$, where the cross section for $p-p \rightarrow \pi$ collisions is $\sigma_{\mathrm{pp}}=3 \times 10^{-26} \mathrm{~cm}^{2}, K=0.5$ is the in-elasticity coefficient, and $\gamma_{\mathrm{p}}$ is the proton Lorentz factor. The energy loss time scale can be expressed by, $\tau_{\mathrm{pp}}=m_{\mathrm{p}} \gamma_{\mathrm{p}} / \dot{P}_{\mathrm{pp}} \approx$ $200 \chi^{5} B_{6}^{6 / 7} M_{17}^{-10 / 7} \gamma_{p} \quad$ s. Before efficient interactions in the acceleration region, protons may be convected onto the WD surface with the accreting matter. The characteristic free fall time scale from the distance of the Alfven radius is, $\tau_{\mathrm{f}}=R_{\mathrm{A}} / v_{\mathrm{f}} \approx 13 \chi^{3 / 2} B_{6}^{6 / 7} M_{17}^{-3 / 7}$ s. We assume that the maximum energies of accelerated protons are determined by their convection out of the transition region, i.e. $\chi$ is not far from 1 . By comparing the energy gain time scale, $\tau_{\mathrm{acc}}=m_{\mathrm{p}} \gamma_{\mathrm{p}} / \dot{P}_{\mathrm{acc}}$, with the escape time scale, $\tau_{\mathrm{f}}$, we estimate the maximum Lorentz factors of accelerated protons, $\gamma_{\mathrm{p}} \approx 1.4 \times 10^{4} \beta \chi^{-5 / 2} B_{6}^{-3 / 7} M_{17}^{5 / 7}$. Protons with such Lorentz factors produce neutral pions which decay to two $\gamma$-ray photons. The characteristic energies of these photons can be estimated on $E_{\gamma} \approx(K / 2 \mu) m_{\mathrm{p}} \gamma_{\mathrm{p}} \approx 165 \beta \chi^{-5 / 2} B_{6}^{-3 / 7} M_{17}^{5 / 7} \mathrm{GeV}$. where $\mu\left(\mu \approx 2.57 \log \left(2 \gamma_{p}\right)-6.45\right.$ for $\gamma_{\mathrm{p}}>>1$, see Orth \& Buffington 1976) is the multiplicity of pions produced by protons. On the other hand, charged pions produced by protons decay into leptons which Lorentz factors are of the order of, $\gamma_{\mathrm{e}}^{\mathrm{sec}} \approx(K / 4 \mu)\left(m_{\mathrm{p}} / m_{\mathrm{e}}\right) \gamma_{\mathrm{p}}$. Leptons with such energies produce synchrotron photons in the magnetic field on the WD surface with characteristic energies, $\varepsilon_{\max }^{\mathrm{sec}} \approx m_{\mathrm{e}}\left(B_{\mathrm{WD}} / B_{\mathrm{cr}}\right)\left(\gamma_{\mathrm{e}}^{\mathrm{sec}}\right)^{2} \approx$ $38 \beta^{2} \chi^{-5} B_{6}^{1 / 7} M_{17}^{10 / 7} \mathrm{MeV}$. For likely parameters, these synchrotron spectra can extend even up to the Fermi-LAT energy range.

In our scenario $\gamma$-rays are produced relatively close to the surface of the WD in the accretion column where the density of matter is large and radiation field from the whole WD surface and the accretion column is strong. We consider the escape conditions of $\gamma$-ray photons from such a radiation field. The UV observations of the WDs allows to estimate the characteristic temperature on their surface in the range $T_{\mathrm{WD}}=(15-25) \times 10^{3} \mathrm{~K}$. We estimate the optical depths for $\gamma$-rays in the radiation field from the whole surface of the $\mathrm{WD}, \tau_{\gamma \gamma}^{\mathrm{WD}} \approx R_{\mathrm{WD}} / \lambda_{\gamma \gamma} \approx R_{\mathrm{WD}} n_{\mathrm{WD}} \sigma_{\gamma \gamma \rightarrow \mathrm{e}^{ \pm}} \approx 0.02 T_{4}$, where $n_{\mathrm{WD}}$ is density of thermal photons close to the surface of the WD with the characteristic temperature $T_{\mathrm{WD}}=2 \times 10^{4} T_{4} \mathrm{~K}, \lambda_{\gamma \gamma}$ is the mean free path for $\gamma \gamma \rightarrow e^{ \pm}$absorption process, and 
$\sigma_{\gamma \gamma \rightarrow \mathrm{e}^{ \pm}}$is the maximum cross section for this process. Based on the above estimates, we conclude that $\gamma$-rays produced close to the surface of the WD escape without significant absorption through the thermal radiation field.

The maximum power available for the acceleration of particles is limited by the energy extracted at the transition region. This energy can be supplied by two mechanisms. In the case of a quasi-spherical accretion from the stellar wind, the matter has to be accelerated to the velocity of the rotating magnetosphere at $R_{\mathrm{A}}$ or to the keplerian velocity. The rotating WD decelerates, providing energy to the turbulent region. In the case of accretion through the Lagrangian point, the matter has a large angular momentum, which has to be partially lost in the transition region in order to guarantee the accretion process up to the WD surface. The angular momentum of the accreting matter is then supplied partially to the transition region and to the WD. As a result, the WD can reach the angular momentum and accelerates.

In the case of the accretion process occurring through the accretion disk, matter arrives to the transition region with the Keplerian velocity. In order to accrete onto the WD surface, the matter from the disk has to be slowed down to the rotational velocity of the WD magnetosphere, i.e. from $v_{\text {kep }}$ to $v_{\text {rot }}$. Then, the maximum available power extracted in the transition region is, $L=$ $\frac{1}{2} \dot{M}_{\text {acc }}\left|v_{\mathrm{k}}^{2}-v_{\text {rot }}^{2}\right|$, which for the accretor phase is, $L \approx 1.7 \times 10^{33} \chi^{-1} B_{6}^{-4 / 7} M_{17}^{9 / 7} \mathrm{erg} \mathrm{s}^{-1}$. provided that $v_{\mathrm{k}}>>v_{\text {rot }}$ at the Alfven radius. We assume that a part, $\delta$, of this energy reservoir is transferred to relativistic electrons and protons, $\delta_{\mathrm{e}}$ and $\delta_{\mathrm{p}}$, respectively. The ratio of $\delta_{\mathrm{e}} / \delta_{\mathrm{p}}$ can not be at present reliably determined by any theory of particle acceleration.

\section{X-rays and $\gamma$-rays from V1223 Sgr}

V1223 Sgr belongs to the class of so called Intermediate Polar White Dwarfs. V1223 Sgr accretes at a relatively large rate, estimated on $\dot{M} \approx 10^{17} \mathrm{~g} \mathrm{~s}^{-1}$ which is derived from the observed X-ray luminosity $L_{\mathrm{X}}=2 \times 10^{34} \mathrm{erg} \mathrm{s}^{-1}$ (e.g. Barlow et al. 2006, Revnivtsev et al. 2008), assumed WD radius $4.17 \times 10^{8} \mathrm{~cm}$ and its mass $1.17 M_{\odot}$ (see Beuermann et al. 2004). The distance to this source is estimated on $510 \mathrm{pc}$. The White Dwarf with the rotational period of $745.5 \mathrm{~s}$ is on the orbit with the period $3.366 \mathrm{hr}$ around the low mass companion. The surface magnetic field of the WD is estimated in the range $(0.5-8) \times 10^{6} \mathrm{G}$, depending on the model (see Beuermann et al. 2004).

We estimate penetration parameter into the WD magnetosphere, $\chi$, by applying the parameters of the accretion scenario for V1223 Sgr derived by Beuermann et al. (2004, see their model A). We estimate value of $\chi \approx 0.4$ based on the comparison of the derived inner radius of the accretion disk. The other parameters of the accretion scenario are the following: $M_{17}=3, B_{6}=0.5$, in order to be consistent with the low energy observations of this binary system (see Beuermann et al. 2004).

We assume that electrons and protons are accelerated in the turbulent transition region with a power-law spectrum characterised by the exponential cut-off. As shown above, electrons lose energy on different radiation processes. The most important are the synchrotron process (dominates at the highest energies) and the ICS of thermal radiation from the WD surface. Protons are likely convected onto the WD surface with the accreting matter and produce pions in collisions with matter. The spectra of $\gamma$-rays (from decay of $\pi^{\circ}$ ) and secondary leptons (from decay of $\pi^{ \pm}$) are calculated assuming the pion multiplicity production in hadronic interactions and the complite colling of protons on the WD surface. 

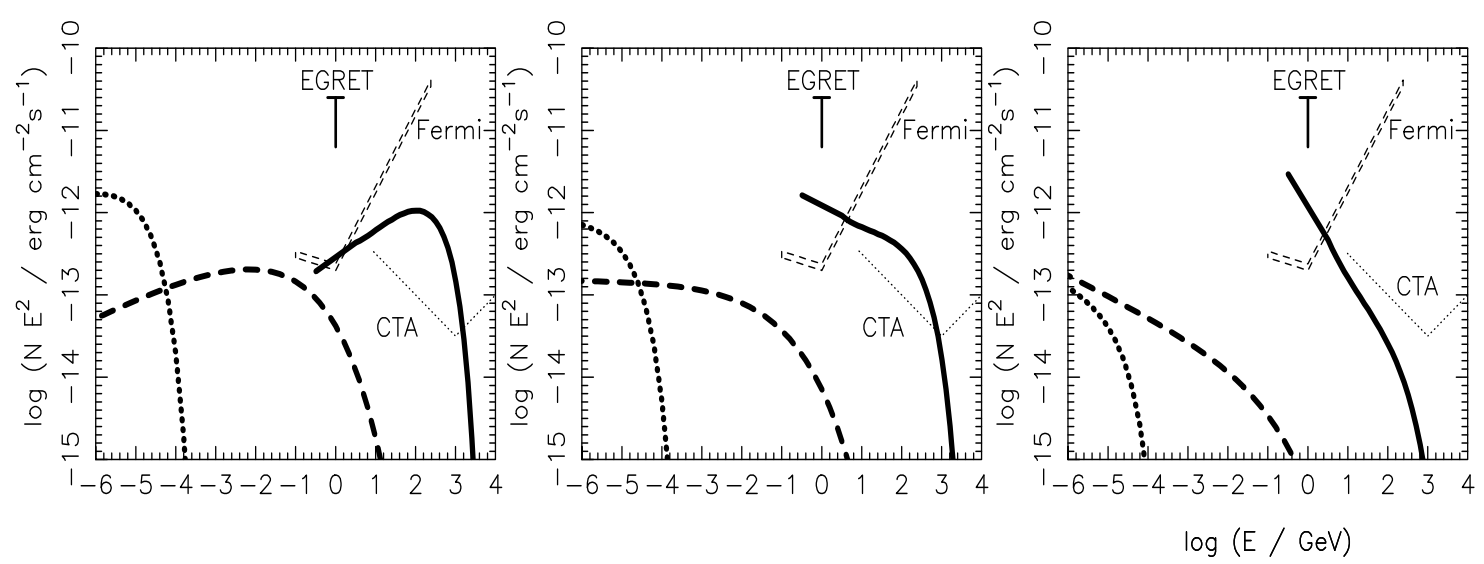

Figure 1: X-ray and $\gamma$-ray spectra expected from the intermediate polar Cataclysmic Variable V1223 Sgr. The spectra are produced by primary electrons in the synchrotron process (dotted curve), secondary leptons from decay of charged pions also in the synchrotron process (dashed) and directly from decay of neutral pions (solid). Pions are produced by protons in hadronic collisions with matter close to the WD surface. Specific figures show the photon spectra for differential spectrum of the primary particles with the spectral index -1.5 (left figure), -2. (middle), and -2.5 (right). The energy conversion coefficients from the acceleration region into the relativistic primary electrons and protons are equal to $\delta_{\mathrm{e}}=\delta_{\mathrm{p}}=0.1$. The parameters describing the WD in V1223 Sgr and the acceleration scenario are reported in the main text. The thin dashed and dotted lines mark the sensitivities of the Fermi-LAT telescope and the future CTA Observatory, respectively. The EGRET upper limit on the $\gamma$-ray emission from V1223 Sgr is marked by the solid arrow.

The results of calculations of the X-ray spectra from synchrotron process of primary electrons, $\mathrm{X}$-rays from synchrotron process of secondary leptons and $\gamma$-rays from decay of neutral pions for different spectral indices of accelerated particles are shown in Fig. 1 The synchrotron emission from secondary leptons extends through the soft $\gamma$-ray energy range and is close to the sensitivity limit of the Fermi-LAT detector. The GeV-TeV $\gamma$-ray emission from decay of neutral pions has been calculated for energies above $300 \mathrm{MeV}$ where the sensitivity of the Fermi-LAT telescope is optimal. This emission could be visible by the Fermi-LAT and/or Cherenkov telescopes depending on the spectral index of primary particles and the efficiency of particle acceleration. There is a chance that the second stages of the H.E.S.S. and the MAGIC arrays (the H.E.S.S. II and MAGIC II) will detect some $\mathrm{CV}$ s of the IP type in the case of extensive observations at the sub-TeV energies.

\section{Discussion and Conclusion}

Considered here model predicts also $\gamma$-ray emission from decay of neutral pions produced in hadronic collisions in the case of V1223 Sgr which is one of the strongest X-ray emitters among the Intermediate Polar type of Cataclysmic Variables (see for details Fig. 1). Depending on the spectral index of hadrons accelerated in the transition region, this $\gamma$-ray emission can be detected by the Fermi-LAT telescope and/or the future CTA observatory. At present, only Fermi-LAT telescope has enough sensitivity to detect IPCVs in the most optimistic case and so constrain the values of the free parameters of the model. In the optimal conditions, V1223 Sgr (and similar sources) have a chance to be even detected by the extensive low threshold $(\sim 100 \mathrm{GeV})$ observations with 
the next generation of the Cherenkov telescope arrays such as H.E.S.S. II and MAGIC II. In the case of steep spectra of accelerated hadrons, the LAT telescope should be able to detect a signal from V1223 Sgr, provided that the efficiency of energy conversion from the transition region to the relativistic hadrons is equal to (or above) the applied value of $10 \%$. Up to now, none of the Cataclysmic Variables has been identified in the error boxes of the Fermi-LAT source catalogue (Abdo et al. 2010). We suggest the analysers to make a closer search for the CVs in the error boxes of the unidentified Fermi-LAT sources. Note that there are several similar to V1223 Sgr Intermediate Polar WDs in both hemispheres (see Tables in Barlow et al. 2006; Revnivtsev et al. 2008) which might be potentially detected in the $\gamma$-rays.

\section{References}

[1] Abdo, A.A. et al. 2010 ApJS 188, 405

[2] Barlow, E.J. et al. 2006 MNRAS 372, 224

[3] Bednarek, W. 2009 A\&A 495, 919

[4] Beuermann, K., Harrison, Th. E., McArthur, B.E., Benedict, G.F., Gänsicke, B.T. 2004 A\&A 419, 291

[5] Bhat, C.L. et al. 1991 ApJ 369, 475

[6] Bowden, C.C.G. et al. 1992 Aph 1, 47

[7] Chadwick, P.M. et al. 1995 Aph 4, 99

[8] Lang, M.J. et al. 1998 Aph 9, 203

[9] Meintjes, P.J. et al. 1992 ApJ 401, 325

[10] Meintjes, P.J. et al. 1994 ApJ 434, 292

[11] Orth, C.D., Buffington, A. 1976 ApJ 206, 312

[12] Revnivtsev, M., Sazonov, S., Krivonos, R., Ritter, H., Sunyaev, R. 2008 A\&A 489, 1121

[13] Schlegel, E.M., Barrett, P.E., de Jager, O.C., Chanmugam, G., Hunter, S., Mattox, J. 1995 ApJ 439, 322

[14] Shakura,N.I., Sunyaev, R.A. 1973 A\&A 24, 337

[15] Sidro, N. et al. 2008 Proc. 30th ICRC (Merida), eds. Caballero R. et al. v. 2, p. 715

[16] Tavani, M. Liang, E. in Proc. Compton Gamma-Ray Observatory Symp. ed. M. Friedlander, N. Gehrels, D. Macomb (New York: AIP), No. 280, p. 428 